\title{
ANÁLISE DA MASTIGAÇÃO EM INDIVÍDUOS QUE APRESENTAM MORDIDA CRUZADA UNILATERAL NA FAIXA-ETÁRIA DE 07 A 12 ANOS
}

\section{Chewing analysis in subjects aged between 07 to 12 years with unilateral cross bite}

\author{
Silvana da Gama Pastana ${ }^{(1)}$, Simone de Menezes Costa ${ }^{(2)}$ \\ Ana Lúcia de Magalhães Leal Chiappetta ${ }^{(3)}$
}

\section{RESUMO}

Objetivo: analisar a função de mastigação relacionada à mordida cruzada posterior unilateral em crianças na faixa etária de sete a doze anos. Métodos: dez indivíduos de ambos os sexos com mordida cruzada unilateral posterior sem intervenção ortodôntica. Na avaliação fonoaudiológica os itens considerados foram: corte do alimento, lado da mastigação, ritmo, postura labial, escape de alimentos, movimento de mandíbula, participação exagerada da musculatura perioral, acúmulo de alimento no vestíbulo da boca, posição e tamanho do bolo alimentar. Resultados: dos dez indivíduos avaliados, $80 \%$ apresentaram mastigação unilateral do mesmo lado da mordida cruzada posterior unilateral e $20 \%$ mastigação bilateral. Dos oito indivíduos analisados com mastigação unilateral, os resultados encontrados foram: corte anterior, sem escape de alimentos e movimentos rotatórios $100 \%$; ritmo lento $50 \%$ e rápido $50 \%$; lábios fechados 75\%; participação exagerada da musculatura perioral 62,5\%; sem acúmulo de alimentos $87,5 \%$; bolo alimentar centralizado $75 \%$; tamanho do bolo pequeno $62,5 \%$. Conclusão: dos casos avaliados, observou-se que $80 \%$ confirmam a relação entre mastigação unilateral e mordida cruzada posterior unilateral. Na mastigação, não foram evidenciadas quaisquer outras alterações que possam estar relacionadas à mordida cruzada posterior unilateral e mastigação unilateral.

DESCRITORES: Oclusão Dentária; Má Oclusão; Força de Mordida; Mastigação; Sistema Estomatognático

\section{INTRODUÇÃO}

A mastigação é a função mais importante do sistema estomatognático. Tem por objetivo a de-

(1) Fonoaudióloga da Prefeitura do Rio de Janeiro e da Universidade do Estado do Rio de Janeiro; Especialização em Motricidade Orofacial pelo CEFAC - Saúde e Educação.

(2) Fonoaudióloga da Prefeitura do Rio de Janeiro e do Hospital Estadual Adão Pereira Nunes; Especialização em Motricidade Orofacial pelo CEFAC - Saúde e Educação.

(3) Fonoaudióloga do Setor de Investigação em Doenças Neuromusculares da Universidade Federal de São Paulo; Doutora em Neurociências pela Universidade Federal de São Paulo. gradação mecânica dos alimentos ${ }^{1}$ e influencia diretamente no crescimento e desenvolvimento dentofacial $^{2}$.

A mastigação bilateral alternada é a mastigação fisiológica e ideal do ser humano, com ciclos mastigatórios tanto de um lado como do outro, e com a presença de movimentos rotatórios de mandíbula ${ }^{3}$, sendo realizada com os lábios ocluídos, é apontada como padrão maduro de mastigação ${ }^{4}$. Este padrão de mastigação possibilita a distribuição da força mastigatória intercalando trabalho e repouso; promove a sincronia e equilíbrio muscular e funcional ${ }^{5}$; estimula o desenvolvimento e/ou manutenção dos arcos dentários e a estabilidade oclusal ${ }^{1}$. 
A mastigação unilateral é o padrão de mastigação em que se observa que a trituração e a pulverização do alimento ocorrem exclusiva ou predominantemente em um dos lados da cavidade oral ${ }^{4}$. Neste padrão ocorre estímulo inadequado do crescimento dentofacial; maior desenvolvimento da mandíbula do lado do balanceio e maior desenvolvimento da maxila do lado do trabalho ${ }^{4-7}$.

A mordida cruzada posterior implica numa atresia do arco dentário superior, de origem esquelética ou dento-alveolar, com desvio mandibular ${ }^{8-10}$ influenciando o padrão de fechamento da mandíbula ${ }^{11}$. A relação entre mordida cruzada posterior unilateral e mastigação unilateral, pode ser explicada pela Lei da Mínima Dimensão Vertical e Ângulo Funcional Mastigatório de Planas. Esta Lei afirma que, partindo-se da oclusão cêntrica, ao deslizar a mandíbula à direita e à esquerda contatando os dentes, o lado aonde houver o menor aumento do terço inferior da face é o lado que mastiga ${ }^{2}$. Na mordida cruzada unilateral há uma diminuição do espaço vertical e a impossibilidade de realizar o movimento de balanceio deste lado, levando o indivíduo a mastigar do lado cruzado ${ }^{12,13}$.

O objetivo desta pesquisa é analisar a função de mastigação relacionada à mordida cruzada posterior unilateral, procurando obter dados que ratifiquem esta unilateralidade e, ainda, observar possíveis alterações que estejam associadas nesta relação.

\section{MÉTODOS}

Foram avaliados 25 indivíduos entre sete e doze anos. Foram excluídos da pesquisa indivíduos com alterações neurológicas, cognitivas, psicológicas e outras alterações estruturais, sendo considerados somente, indivíduos com diagnóstico odonto-lógico exclusivo de mordida cruzada unilateral posterior. Após aplicação do critério de exclusão, dez indivíduos foram selecionados, sendo quatro do sexo feminino e seis do masculino. Todos estão em processo de avaliação fonoau-diológica e/ou odontológica, sem intervenção ortodôntica, sendo três da Clínica de Fonoau-diologia da Faculdade de Odontologia da Universidade do Estado do Rio de Janeiro (UERJ) e sete do Posto de Atendimento Médico Maria Cristina Roma Paugartten da Prefeitura do Rio de Janeiro.

$\mathrm{Na}$ avaliação da mastigação os dados foram coletados através de protocolo adaptado do Setor de Fonoaudiologia da Faculdade de Odontologia da UERJ (Figura 1). Os itens considerados foram: corte do alimento, lado da mastigação, ritmo, postura labial, escape de alimentos, movimento de mandíbula, participação exagerada da musculatura perioral, acúmulo de alimento no vestíbulo da boca, posicionamento do bolo alimentar e tamanho do bolo alimentar. A avaliação foi realizada por duas examinadoras devidamente calibradas. Foi utilizado na avaliação da mastigação o pão francês, que é um alimento que favorece a visualização desta função e que, principalmente, não causa atipias erroneamente, sendo um alimento de baixo custo, fácil aquisição e de boa aceitação por grande parte dos pacientes ${ }^{14}$. Todos os indivíduos foram filmados, fotografados e avaliados nos consultórios fonoaudiológicos de cada unidade envolvida na pesquisa.

Os dados foram analisados por meio de distribuição de freqüência absoluta e relativa.

A presente pesquisa foi avaliada e aprovada pelo Comitê de Ética em Pesquisa do Centro de Especialização em Fonoaudiologia Clínica, sob $\mathrm{n}^{\circ} 086 / 05$, tendo sido considerada como sem risco e com necessidade do consentimento livre e esclarecido.

\section{RESULTADOS}

De acordo com a avaliação realizada, oito $(80 \%)$ dos dez indivíduos participantes da pesquisa apresentaram mastigação unilateral do mesmo lado da mordida cruzada posterior e dois $(20 \%)$ apresentaram mastigação bilateral, conforme demonstrado na Tabela 1.

Do total de oito $(80 \%)$ indivíduos com mordida cruzada posterior unilateral e mastigação unilateral, foram observados os seguintes resultados: $100 \%$ dos oito indivíduos avaliados, nesta amostra, apresentaram corte anterior do alimento, movimentos rotatórios de mandíbula e nenhum escape do alimento; quanto ao ritmo mastigatório quatro $(50 \%)$ apresentaram ritmo lento e quatro $(50 \%)$ ritmo rápido; seis $(75 \%)$ mantiveram os lábios fechados e dois $(25 \%)$ abertos; cinco $(62,5 \%)$ indivíduos demonstraram participação exagerada da musculatura perioral e três $(37,5 \%)$ não; não foi observado acúmulo de alimentos em sete $(87,5 \%)$ indivíduos e um $(12,5 \%)$ apresentou; a posição do bolo na preparação para a deglutição foi centralizado em seis $(75 \%)$ indivíduos e em dois (25\%) não foi centralizado; cinco $(62,5 \%)$ indivíduos apresentaram tamanho do bolo alimentar pequeno e três $(37,5 \%)$ apresentaram tamanho grande, conforme demonstrado na Tabela 2. 


\section{PROTOCOLO}

Protocolo de avaliação da função de mastigação adaptado do Setor de Fonoaudiologia da Faculdade de Odontologia da Universidade do Estado do Rio de Janeiro - UERJ

DATA:

NOME

DATA DE NASC:

1-Número de dentes:

Em cima à dircita:

Em baixo à direita:

Total: dade atual:

Em cima à esquerda:

Em baixo à esquerda:

2- Tipo de dentição:

( ) decidua ( ) mista ( ) permanente

3- Classificar o tipo de oclusão.

Mordida cruzada unilateral: ( ) Sim ( ) Não

Se sim: D( ) E()

\section{EXAME DA FUNÇÃO DE MASTIGAÇÃO}

4- Mastigação:

corte do alimento:

( ) com os dentes anteriores ( ) com os dentes laterais tipo:

( ) bilateral ( ) unilateral D ( ) unilateral

ritmo:

( ) lento ( ) rápido

lábios:

( ) fechados ( ) abertos

escape de alimentos na comissura dos lábios:

( ) Não ( ) Sim

movimentos de mandibula:

( ) rotatórios ( ) verticais

participação exagerada da musculatura perioral:

( ) Não ( ) Sim

acúmulo de alimento no vestibulo da boca

( ) Não ( ) Sim

posicionamento do bolo alimentar

( ) centralizado ( ) não centralizado

tamanho do bolo alimentar

( ) pequeno ( )grande

Figura 1 - Protocolo de avaliação da função de mastigação adaptado do Setor de Fonoaudiologia da Faculdade de Odontologia da Universidade do Estado do Rio de Janeiro - UERJ

Tabela 1 - Distribuição dos tipos de mordida cruzada posterior e mastigação e a relação de unilateralidade

\begin{tabular}{c|cc|cccc}
\hline & \multicolumn{2}{c}{$\begin{array}{c}\text { Mordida Cruzada } \\
\text { Posterior }\end{array}$} & \multicolumn{2}{c|}{ Mastigação } & \multicolumn{2}{c}{$\begin{array}{c}\text { Mordida e Mastigação } \\
\text { do Mesmo Lado }\end{array}$} \\
& $\mathrm{N}$ & $\%$ & $\mathrm{~N}$ & $\%$ & $\mathrm{~N}$ & $\%$ \\
\hline Unilateral Direita & 4 & 40 & 3 & 30 & 3 & 30 \\
\hline Unilateral Esquerda & 6 & 60 & 5 & 50 & 5 & 50 \\
\hline Bilateral & - & - & 2 & 20 & - & - \\
\hline Total & 10 & 100 & 10 & 100 & 8 & 80 \\
\hline
\end{tabular}


Tabela 2 - Achados na avaliação da mastigação unilateral na Mordida Cruzada Posterior Unilateral

\begin{tabular}{lllllll}
\hline \multicolumn{1}{c}{ Itens Avaliados } & Respostas & $\mathrm{N}$ & $\%$ & Respostas & $\mathrm{N}$ & $\%$ \\
\hline 1. Corte do Alimento & Dentes anteriores & 8 & 100 & Dentes laterais & 0 & 0 \\
\hline 2. Ritmo & Lento & 4 & 50 & Rápido & 4 & 50 \\
\hline 3. Lábios & Fechados & 6 & 75 & Abertos & 2 & 25 \\
\hline 4. Escape de alimentos & Não & 8 & 100 & Sim & 0 & 0 \\
\hline 5. Movimentos de mandibula & Rotatórios & 8 & 100 & Verticais & 0 & 0 \\
\hline $\begin{array}{l}\text { 6. Participaçäo exagerada dal } \\
\text { musculatura perioral }\end{array}$ & Não & 3 & 37,5 & Sim & 5 & 62,5 \\
\hline 7. Acúmulo de alimento em vestibulo & Não & 7 & 87,5 & Sim & 1 & 12,5 \\
\hline 8. Posiçäo do bolo alimentar & Centralizado & 6 & 75 & Não centralizado & 2 & 25 \\
\hline 9. Tamanho do bolo alimentar & Pequeno & 5 & 62,5 & Grande & 3 & 37,5 \\
\hline
\end{tabular}

\section{DISCUSSÃo}

A intenção desta pesquisa foi a de observar a ocorrência da mastigação unilateral e outras possíveis alterações relacionadas a esta e à mordida cruzada posterior unilateral e ratificar a unilateralidade da forma e da função.

Sabe-se que, o padrão ideal de mastigação é o bilateral alternado que permite a distribuição de força mastigatória, alternando trabalho e repouso muscular e articular ${ }^{5}$, proporcionando adequado desenvolvimento, crescimento e estabilidade das arcadas dentárias ${ }^{4}$. Será responsável também, pelo desenvolvimento de ossos, maxilas, manutenção dos arcos, estabilidade da oclusão e pelo equilíbrio muscular e funcional ${ }^{15}$, sendo essencial na prevenção dos distúrbios miofuncionais, pois estimula a musculatura orofacial contribuindo para a harmonia facial ${ }^{1,16-19}$, sendo portanto, de grande importância no crescimento crânio facial ${ }^{7}$.

Já a mastigação unilateral, promove o estímulo inadequado do crescimento e da estabilização das estruturais orofaciais, proporcionando uma excitação neural, que terá como conseqüência um maior desenvolvimento da mandíbula do lado do balanceio e um maior desenvolvimento da maxila do lado do trabalho ${ }^{2}$. A mastigação unilateral causará uma série de desvios, que poderão acarretar alterações de ordem morfológicas em todo o sistema mastigatório ${ }^{20}$.

A mordida cruzada posterior é uma alteração transversal que se caracteriza pela inversão da oclusão, ou seja, os dentes superiores se posicionam no sentido vestíbulo lingual em relação aos inferiores, reduzindo assim a dimensão transversal da maxila ${ }^{21,22}$.

A mastigação unilateral associada à mordida cru- zada posterior unilateral pode ser explicada pela Lei da Mínima Dimensão Vertical de Planas e diz que, partindo-se da oclusão cêntrica, ao deslizar a mandíbula à direita e à esquerda contatando os dentes, o lado onde houver o menor aumento do terço inferior da face, é o lado que mastiga ${ }^{2,23}$. As estruturas do sistema estomatognático, tendem a adaptar seus padrões de funcionalidade na presença de uma alteração ${ }^{24}$, como forma de prevenir traumas no periodonto, dentes e articulações ${ }^{4,5}$.

Apesar dos achados não terem demonstrado valores significantes nesta amostra, faz-se necessárias algumas considerações.

A relação da mastigação unilateral e da mordida cruzada posterior unilateral, corrobora com o que a literatura afirma $^{2-5,12,13,17,24-29}$. O que se pôde perceber foi que, nos oito (80\%) indivíduos analisados com mastigação unilateral, isto ficou bastante evidente, já que, todos apresentaram a mastigação exclusiva do mesmo lado da mordida cruzada, confirmando com isso, a relação de unilateralidade em $80 \%$ da amostra. Nos dois (20\%) restantes, foi evidenciada mastigação do tipo bilateral. Há estudo que comprova a ocorrência da bilateralidade da função mastigatória, em indivíduos com mordida cruzada posterior unilateral ${ }^{30}$.

$\mathrm{Na}$ observação dos aspectos funcionais da mastigação unilateral associada à mordida cruzada posterior unilateral, o que se pôde perceber foi que, todos os oito (100\%) indivíduos avaliados com mastigação unilateral apresentaram corte anterior do alimento, movimentos rotatórios de mandíbula e não apresentaram escape do alimento. Tal unanimidade sugere que, a relação de unilateralidade da função de mastigação e da forma da mordida, nesta amostra, não afeta o modo de apreen- 
são, o controle do bolo e os movimentos rotatórios da mandíbula do lado da mordida.

Em relação ao ritmo, quatro (50\%) apresentaram ritmo lento e outros quatro $(50 \%)$ realizaram a mastigação de forma rápida. Uma lentidão mastigatória pode estar associada às disfunções da articulação temporomandibular e a movimentos mandibulares limitados, uma vez que, a mordida cruzada posterior unilateral e/ou mastigação unilateral podem levar a estas disfunções ${ }^{4-6,9,10,27,28,31-33}$. Amastigação rápida geralmente reflete poucos ciclos mastigatórios, levando a uma trituração e pulverização ineficiente do alimento, sendo este engolido em pedaços, o que pode ser decorrente da respiração oral ou oronasal ${ }^{33}$, falta de propriocepção e hipofunção da musculatura mastigatória ${ }^{4}$. Dos quatro $(50 \%)$ indivíduos com ritmo rápido de mastigação, três $(37,5 \%)$ também apresentaram tamanho de bolo grande e ainda, participação da musculatura perioral. Um volume grande de alimento levará a uma maior contração da musculatura perioral ${ }^{33}$.

A participação da musculatura perioral, foi observada em cinco $(62,5 \%)$ indivíduos, o que geralmente revela uma mastigação inadequada e é apresentada em indivíduos de face longa ${ }^{5,12}$ com postura de lábios abertos ou entreabertos na postura habitual de repouso ou de respiração oral ou oronasal $4,24,34,35$.

Dois (20\%) indivíduos apresentaram mastigação sem vedamento labial, tal postura funcional pode estar ligada a vários fatores como: respiração oral ou oronasal; hipofunção da musculatura elevadora da mandíbula, lábios abertos em postura habitual; musculatura flácida de lábios e bochechas; lábios superior retraído e/ou inferior com eversão e falta de propriocepção ${ }^{4} \mathrm{e}$, ainda, o volume do alimento ${ }^{19}$.

Somente um $(12,5 \%)$ indivíduo apresentou várias alterações; além do acúmulo de alimento em ves- tíbulo; falta de vedamento labial; ritmo rápido; participação exagerada da musculatura perioral; bolo alimentar descentralizado e grande. Do total de oito $(100 \%)$ indivíduos, sete $(87,5 \%)$ não apresentaram acúmulo de alimento em vestíbulo. Isso pode ser explicado pela atividade aumentada, pois ocorre uma maior potência muscular do lado do trabalho ${ }^{5}$, desse modo, o músculo bucinador no lado da mordida, encontra-se em contração excessiva devido à mastigação unilateral. A função do músculo bucinador é de reconduzir o alimento para as faces oclusais e centro da cavidade oral ${ }^{33,35}$. Quando existe uma mordida cruzada, a curva de Wilson ${ }^{36}$ encontra-se alterada, conseqüentemente o alimento dirige-se mais para o vestíbulo, fazendo com que o músculo bucinador trabalhe excessivamente ${ }^{5,37}$.

Não foram encontrados estudos que fizessem relação da mordida cruzada posterior unilateral e aos aspectos referentes à função mastigatória do mesmo lado da mordida. As alterações encontradas nesta amostra, não são características da associação da mordida cruzada posterior unilateral e mastigação unilateral e, podem estar relacionadas com vários outros fatores, conforme verificado na literatura ${ }^{4-6,9,10,12,19,24,27,29,31-35,37 . ~}$

\section{CONCLUSÃO}

Houve confirmação da relação de unilateralidade entre mastigação e mordida cruzada posterior. Dos itens observados na mastigação, não foram evidenciadas quaisquer outras alterações que possam estar relacionadas à mordida cruzada posterior unilateral e mastigação unilateral, já que não houve significância quanto aos resultados e as alterações encontradas podem ser decorrentes de vários fatores.

\begin{abstract}
Purpose: to analyze the function of chewing, related to the posterior unilateral crossbite in children aged between 07 to 12 years. Methods: 10 samples of both genders with posterior unilateral crossbite, with no orthodontic treatment history. During the speech language pathology evaluation the examined items were: food cutting, side of the chewing, rhythm, lips position, food escape, jaw movement, exaggerated participation of the perioral muscles, food accumulation in the vestibule of the mouth, position and size of the alimentary cake. Results: from the 10 evaluated samples, $80 \%$ demonstrated unilateral chewing of the same side of the poste-rior unilateral crossbite and $20 \%$ bilateral chewing. From the 08 analyzed samples, the results were: previous cut, without escape of food and rotatory movements 100\%; slow rhythm 50\% and fast rhythm 50\%; closed lips $75 \%$; exaggerated participation of perioral muscles $62,5 \%$; without accumulation of food $87,5 \%$; alimentary cake cente-red $62,5 \%$; small cale size $62,5 \%$. Conclusion: from the evaluated cases, it was observed that $80 \%$ confirmed the relation between unilateral chewing and unilateral posterior crossbite, corroborating the literature's reporting. In the chewing, no any alterations related to the posterior unilateral crossbite and unilateral chewing have been noted.
\end{abstract}

KEYWORDS: Dental Occlusion; Malocclusion; Bite Force; Mastication; Stomatognathic System 


\section{REFERÊNCIAS}

1. Douglas CR. Patofisiologia oral. São Paulo: Pancaster; 1998.

2. Planas P. Reabilitação neuro-oclusal. Rio de Janeiro: Médici; 1997.

3. Amaral DB. Mastigação unilateral x oclusão normal: um estudo sobre sua ocorrência em crianças de 4 a 5 anos. Rev CEFAC. 2000; 2(2):23-30.

4. Cattoni DM. Alteração da mastigação e deglutição. In: Ferreira LP, Befi-Lopes DM, Limongi SCO.Tratado de fonoaudiologia. São Paulo: Roca; 2004. p. 277-84. 5. Bianchini EMG. Mastigação e ATM. In: Marchesan IQ. Fundamentos em fonoaudiologia: aspectos clínicos da motricidade oral. Rio de Janeiro: GuanabaraKoogan; 2005. p. 46-57.

6. Pinto AS, Buschang PH, Throckmorton GS, Chen P. Morphological and positional asymmetries of young children with functional unilateral posterior crossbite. Am J Orthod Dentofacial Orthop. 2001; 120(5):513-20.

7. Yãnez GR. Funcion muscular em las mordidas cruzadas. Disponível em: URL: http://www.encolombia.com/ ortopeditallado3.htm Acesso em: 14 dez 2005.

8. Brin I, Ben-Bassat Y, Blustein Y, Ehrlich J, Hochman N, Marmary Y, Yaffe A. Skeletal and functional effects of treatment for unilateral posterior crossbite. Am J Orthod Dentofacial Orthop. 1996; 109(2):173-9.

9. Langberg BJ, Arai K, Miner RM. Transverse skeletal and dental asymmetry in adults with unilateral lingual posterior crossbite. Am J Orthod Dentofacial Orthop. 2005; 127(1):6-15.

10. Lam PH, Sadowsky C, Omerza F. Mandibular asymmetry and condylar position in children with unilateral posterior crossbite. Am J Orthod Dentofacial Orthop. 1999; 115(5):569-75.

11. Silva Filho OG, Pinto DM, Álvares LC. Alterações condilares associadas às mordidas cruzadas funcionais. Ortodontia. 1992; 25(2):41-51.

12. Krakauer LRH. Alterações de funções orais nos diversos tipos faciais. In: Marchesan IQ, Bolaff C, Gomes IC et al, organizadores. Tópicos em fonoaudiologia. São Paulo: Lovise; 1995. p. 147-54.

13. Marchesan IQ. The speech pathology treatment with alterations of the stomatognathic system. Int J Orofacial Myology. 2000; 26:5-12.

14. Junqueira P. Avaliação miofuncional. In: Marchesan IQ. Fundamentos em fonoaudiologia: aspectos clínicos da motricidade oral. Rio de Janeiro: GuanabaraKoogan; 2005. p. 19-27.

15. Tanigute CC. Desenvolvimento das funções estomatognáticas. In: Marchesan IQ. Fundamentos em fonoaudiologia: aspectos clínicos da motricidade oral. Rio de Janeiro: Guanabara-
Koogan; 2005. p. 1-9.

16. Pizzol KEDC. Influência da mastigação unilateral no desenvolvimento da assimetria facial. Rev Uniara. 2004; 15:215-9.

17. Gonzáles NZT. Funções orofaciais. In: Gonzáles NZT, Lopes LD. Fonoaudiologia e ortopedia maxilar na reabilitação orofacial: tratamento precoce e preventivo: terapia miofuncional. São Paulo: Santos; 2000. p. 19-27.

18. Gonzáles NZT. Enfoque fonoaudiológico. In: Gonzáles NZT, Lopes LD. Fonoaudiologia e ortopedia maxilar na reabilitação orofacial: tratamento precoce e preventivo: terapia miofuncional. São Paulo: Santos; 2000. p. 39-96.

19. Silva AM, Silva KP, Oliveira RCPS, Ferreira VJA. A influência da alimentação na mastigação em crianças. Fonoaudiol Bras. 2004; 4(1):1-3.

20. Marques Junior JA, Lenci PRJ. Conseqüências da mastigação unilateral no desenvolvimento e equilíbrio do sistema estomatognático. Disponível em: URL: http://www.ortodontiaemrevista.com,br/artigos/mastigunilat.htm Acesso em: 29 set 2005.

21. Felix GB, Jorge TM, Genaro KF. Intervenção fonoaudiológica em pacientes submetidos à cirurgia ortognática. In: Ferreira LP, Befi-Lopes DM, Limongi SCO. Tratado de fonoaudiologia. São Paulo: Roca; 2004. p. 494-511.

22. Marchesan IQ. Motricidade oral: visão clínica do fonoaudiólogo integrado a outras especialidades. São Paulo: Pancast; 1993. p. 70.

23. Santiago Júnior $O$. Incidência da mastigação unilateral em crianças com dentição decídua e dentição mista em estágio inicial com alimentos fibrosos e macios. Rev Fac Odontol Porto Alegre. 1994; 35(1):28-31.

24. Bianchini EMG. Desproporções maxilomandibulares: atuação fonoaudiológica com pacientes submetidos à cirurgia ortognática. In: Marchesan IQ, Bolafi C, Gomes ICD, Zorzi JL. Tópicos em fonoaudiologia. São Paulo: Lovise; 1995. p. 129- 45.

25. Bianchini EMC. Avaliação fonoaudiológica da motricidade oral: anamnese, exame clínico, o quê e por que avaliar. In: Bianchini EMC. Articulação temporo-mandibular: implicações, limitações e possibilidades fonoaudiológicas. São Paulo: Pró-fono; 2000. p. 191-253.

26. Almeida GP. Ortopedia funcional maxilares na dentição decídua Rev Odontol. [periódico online] Disponível em: URL: http://www.iepc.org.br/artigo1.htm Acesso em: 29 set 2005.

27. Throckmorton GS, Buschang PH, Hayasaki $\mathrm{H}$, Pinto AS. Charges in the masticatory cycle following treatment of posterior unilateral crossbite in children. Am J Orthod Dentofacial Orthop. 2001; 120(5):521-9. 28. Miyawaki S, Tanimoto Y, Araki Y, Katayama A, Kuboki T, Yamamoto TT. Movement of the lateral and medial poles of the working condyle during 
mastication in patients with unilateral posterior crossbite. Am J Orthod Dentofacial Orthop. 2004; 126(5):549-54.

29. Kasai MCB, Portella MQ. Intervenção fonoaudiológica em pacientes submetidos ao tratamento ortodôntico cirúrgico. Rev Dental Press Ortod Ortop Facial. 2001; 6(2):79-84.

30. Alarcón JÁ, Martín C, Palma JC. Effect of unilateral posterior crossbite on the electromyographic activity of human masticatory muscles. Am J Orthod Dentofacial Orthop. 2000; 118(3):328-34.

31. Nerder PH, Bakke M, Solow B. The functional shift of the mandible in unilateral posterior crossbite and the adaptation of the temporomandibular joints: a pilot study. Eur J Orthod. 1999; 21(2):155-66.

32. Hesse KL, Artun J, Joondeph DR, Kennedy DB. Changes in condylar position and occlusion associated with maxillary expansion for correction of functional unilateral posterior crossbite. Am J Orthod Dentofacial Orthop. 1997; 111(4):410-8.
33. Felício CM. Fonoaudiologia aplicada a casos odontológicos: motricidade oral e audiologia. São Paulo: Pancast; 1999. p. 31-108.

34. Pereira AC, Jorge TM, Ribeiro Junior PD, Félix GB. Características das funções orais de indivíduos com má oclusão classe III e diferentes tipos faciais. Rev Dental Press Ortod Ortop Facial. 2005; 10(6):111-9.

35. Marchesan IQ. Deglutição-diagnóstico e possibilidades terapêuticas. In: Marchesan IQ. Fundamentos em fonoaudiologia: aspectos clínicos da motricidade oral. Rio de Janeiro: Guanabara-Koogan; 2005. p. 59-66.

36. Rodrigues L. Avaliação odontológica. In: Bianchini EMC. Articulação temporomandibular: implicações, limitações e possibilidades fonoaudiológicas. São Paulo: Pró-fono; 2000. p. 133-64.

37. Dawson PE. Avaliação, diagnóstico e tratamento dos problemas oclusais. São Paulo: Artes Médicas; 1993.

RECEBIDO EM: 23/11/2006

ACEITO EM: 11/07/2007

Endereço para correspondência:

Rua Mariano Sendra dos Santos, 88, ap. 604

Duque de Caxias - RJ

CEP: $25010-080$

Tel: (21) 26716620 / 91825427

E-mail:sgpastana@ig.com.br 